\title{
A case report: Type II Abernethy malformation complicated with congenital polydactyly and enlargement of all cardiac chambers.
}

Cheng Zhu

Shandong University Qilu Hospital

Min Wang ( $\nabla$ doctorminmin@163.com)

Qian Hao

Shandong University Qilu Hospital

\section{Research}

Keywords: Abernethy malformation, congenital polydactyly, differential diagnosis, conservative therapy

Posted Date: December 10th, 2019

DOI: https://doi.org/10.21203/rs.2.18514/v1

License: (a) (i) This work is licensed under a Creative Commons Attribution 4.0 International License. Read Full License

Version of Record: A version of this preprint was published at Open Medicine Journal on September 22nd, 2020. See the published version at https://doi.org/10.2174/1874220302007010032. 


\section{Abstract}

Background: Abernethy malformation is a kind of congenital malformation of portal vein system caused by abnormal portacaval anastomosis. It can be in combination with other congenital malformations. The major therapy of Abernethy malformation is surgery. There has been a limited number of patients since the first patient reported, leading to a limited view towards this kind of disease until now.

Results: In August 2018 we treated a patient diagnosed with typell Abernethy malformation complicated with both congenital polydactyly and enlargement of all cardiac chambers, which is extremely rare and can be a supplementary to the existing cases. Besides, the low white blood cell and platelet, the arrested megakaryocytic maturation and the positive platelet autoantibody in serum may result in misdiagnosis as immune thrombocytopenia, so we analyze the differential points between these two diseases. We treated this patient with silybin orally and advised him to make follow-up visits because of his mild liver function disorder, normal cardiac function and no other malformations or complications complicated. At the latest follow-up, we knew the condition of the patient was generally satisfactory, whether in terms of laboratory test results or his daily life experience.

Conclusions: Because of some changes of spleen in form and function secondary to Abernethy malformation, in some cases, this disease has similarities with a part of blood diseases, which we should take into consideration for differential diagnosis, especially when other congenital malformations are found in combination at the same time. This case also suggests that simply conservative treatment with regular follow-up visits can be suitable for certain patients.

\section{Background}

Abernethy malformation, a rare hepatic vascular malformation, is a kind of congenital malformation of portal vein system caused by abnormal portacaval anastomosis. According to whether the portal vein is absent completely or not, it can be divided into two types. Type区, to which many female and pediatric patients belong, means a total lack of portal vein, patients in this type usually have a short medical history as a result of the rapid progress like liver failure, while patients in type $\mathbb{Z}$ often have a relatively long medical history for its incomplete absence of portal vein[1]. There has been a limited number of patients since the first patient reported by Abernethy in 1797[2], leading to a limited view towards this kind of disease until now. This patient was complicated with congenital polydactyly and enlargement of all cardiac chambers, which is extremely rare and can be a supplementary to the existing cases. Besides, his arrested megakaryocytic maturation and positive platelet autoantibody in serum may result in misdiagnosis as immune thrombocytopenia, which reminds us to take a part of blood diseases into consideration for differential diagnosis.

\section{Patient Information}


We treated the patient in August 2018. His diagnosis is type $\mathbb{A}$ Abernethy malformation complicated with both congenital polydactyly and enlargement of all cardiac chambers. This patient is a 16-year-old boy with a 6-year history of low white blood cell (WBC) and platelet levels. Routine blood tests over the past 6 years showed that his WBC counts ranged from $2.36 \times 10^{9} / \mathrm{L}$ to $3.87 \times 10^{9} / \mathrm{L}$ and his platelets ranged from $48 \times 10^{9} / \mathrm{L}$ to $72 \times 10^{9} / \mathrm{L}$, while those for erythrocytes and hemoglobin were normal. The results of a bone marrow cytology test and bone marrow biopsy in other hospitals showed normal erythroid proliferation, while the granulocytic and megakaryocytic maturation was arrested. The results of fluorescent in situ hybridization (FISH) suggested neither - 7/7q-, the absence of gene EGR1 (5q31) or D20S108, nor increased numbers of chromosome 8. According to our inquiry, his parents and other immediate family were all normal.

\section{Clinical Findings}

After this patient was admitted to our hospital, we gave him basic physical examination and found his spleen was palpated at the bottom of the ribs with a second line 10.0 centimeters long, both of his hands had six fingers and the surplus thumb on the right hand had been resected several years earlier, leaving a small and deformed one (Fig. 1A). A small amount of convex tissue was present on the medial surface of his normal left thumb which was considered to be an incompletely developed finger that was not painful when touched (Fig. 1B). In addition, we gave some basic laboratory examinations for supplementary and the results were as follows: The levels of alanine aminotransferase (ALT) and aspartate transaminase (AST) were both normal, while that of direct bilirubin (11.2umol/L) and indirect bilirubin (27.2umol/L) were elevated slightly. Prothrombin time (PT) was 15.60 s and prothrombin activity was 53.00 percent. Tests for serum GPIb and GPIIb/lla platelet autoantibodies were positive. An abdominal ultrasound showed cavernous transformation of the portal vein (CTPV) and computed tomography angiography (CTA) of the portal vein system showed hepatic hemangioma and obstruction in the main portal vein (MPV), along with collateral circulation development, CTPV, and splenomegaly (Fig. 2A), as well as spleno-gastric fundus venous anastomosis (Fig. 2B) and left spleno-renal venous anastomosis (Fig. 2C). The results of an ultrasonic cardiogram (UCG) showed enlargements of all cardiac chambers, along with a left ventricular ejection fraction (LVEF) of 65\%, $41 \mathrm{~mm}$ diameter left atrium, $52 \times 47 \mathrm{~mm}$ right atrium, $55 \mathrm{~mm}$ left ventricle, and $26 \mathrm{~mm}$ right ventricle. According to the results of abdominal ultrasound and CTA, we took the diagnosis of Abernethy malformation into consideration and give him an indirect portographic examination. During the examination, contrast medium flowed from the splenic vein and superior mesenteric vein to the portal vein in the delayed phase of the examination. Contrast medium was observed in the portal vein closed to the splenic vein, instead of in the intrahepatic segment (Fig. 2D).

\section{Diagnostic Assessment}

In consideration of these symptoms and examination results, the patient was diagnosed with type $\nabla$ Abernethy malformation complicated with congenital polydactyly and enlargement of all cardiac chambers. With regard to this patient, a differential diagnosis with immune thrombocytopenia (ITP) 
should be taken into consideration for the arrested megakaryocytic maturation and the presence of platelet autoantibodies. Although arrested megakaryocytic maturation and platelet autoantibodies can be observed in ITP patients, 70\% of them do not have splenomegaly. Moreover, when diagnosing ITP, secondary thrombocytopenia must be excluded. However, hypersplenism secondary to Abernethy malformation could not be ruled out because the decreased WBCs and platelets may also occur in the early phase of hypersplenism.

\section{Therapeutic Intervention, Follow-up And Outcomes}

Because the patient's liver function was mild disorder, cardiac function was normal despite the enlargement of all cardiac chambers and no malformations or complications associated with other systems were found. We suggested that he simply take silybin orally for a period and make regular follow-up visits. We attained at the latest follow-up that the his platelets were $70 \times 10^{9} / \mathrm{L}$ and WBCs were $3.81 \times 10^{9} / \mathrm{L}$, he did not complain of any discomfort.

\section{Patient Perspective}

This patient is a student with general family economic conditions, the conservative therapy neither keep him from normal study and life, nor bring much financial burden to his family. The treatment effect is also accordance with his and his parents' expected one, they are willing to cooperate with the next followup visits.

\section{Discussion}

Abernethy malformation is a rare congenital vascular malformation that can be in combination with other congenital malformations such as cardiovascular malformations, biliary atresia, choledochus cysts, and polysplenia[3]. This case adds Abernethy malformation with both congenital polydactyly and enlargement of all cardiac chambers to that list. Besides, Abernethy malformation may lead to some changes of spleen in form and function, which can further bring hematological changes. Before this patient was admitted in our hospital, the reason making his laboratory tests abnormal was largely considered to be related to blood diseases. Indeed, Abernethy malformation and a part of blood diseases have some similarites that maybe lead to misdiagnosis easily, but we should attach importance to basic laboratory examinations and not let some results hinder our diagnosis thinking. Last but not least, although the major therapy of Abernethy malformation is surgery, simply conservative treatment with regular follow-up visits can also be suitable for certain patients, this depends on a comprehensive assessment of patients' condition.

\section{Conclusion}

In some cases, Abernethy malformation has similarities with a part of blood diseases, which we should take into consideration for differential diagnosis, especially when other congenital malformations are 
found in combination at the same time. This case also suggests that simply conservative treatment with regular follow-up visits can be suitable for certain patients.

\section{Declarations}

Funding: None received.

Availability of data and materials: Data are available by request.

Ethics approval and consent to participate区Ethical committee of Qi-lu hospital of Shandong University approved our study.

Consent for publication: We have obtained permission from the patient for the purpose of publication, We promise that our team take responsibility for the anonymization of the patient.

Competing interests: We declare that we have no competing interests.

Authors' contributions: Cheng, Z participated in the design and and drafted the manuscript; Min, $\mathrm{W}$ participated in the audit of the manuscript; Qian, $\mathrm{H}$ participated in the data collection and interpretation of the manuscript.

\section{Abbreviations}

WBC: White blood cell

ALT: Alanine aminotransferase

AST: Aspartate transaminase

PT: Prothrombin time

CTPV: Cavernous transformation of the portal vein

CTA: Computed tomography angiography

MPV: Main portal vein

UCG: Ultrasonic cardiogram

LVEF: Left ventricular ejection fraction

ITP: Immune thrombocytopenia

\section{References}


1. Morgan G, Superina R: Congenital absence of the portal vein: Two cases and a proposed classification system for portasystemic vascular anomalies. 29(9):0-1241.

2. Abernethy MJ: Account of Two Instances of Uncommon Formation in the Viscera of the Human Body: From the Philosophical Transactions of the Royal Society of London. 2011, 7:100-108.

3. Singhal A, Srivastava A, Goyal N, Vij V, Wadhawan M, Bera M, Gupta S: Successful living donor liver transplant in a child with Abernethy malformation with biliary atresia, ventricular septal defect and intrapulmonary shunting. Pediatric Transplantation 2009, 13.

\section{Figures}

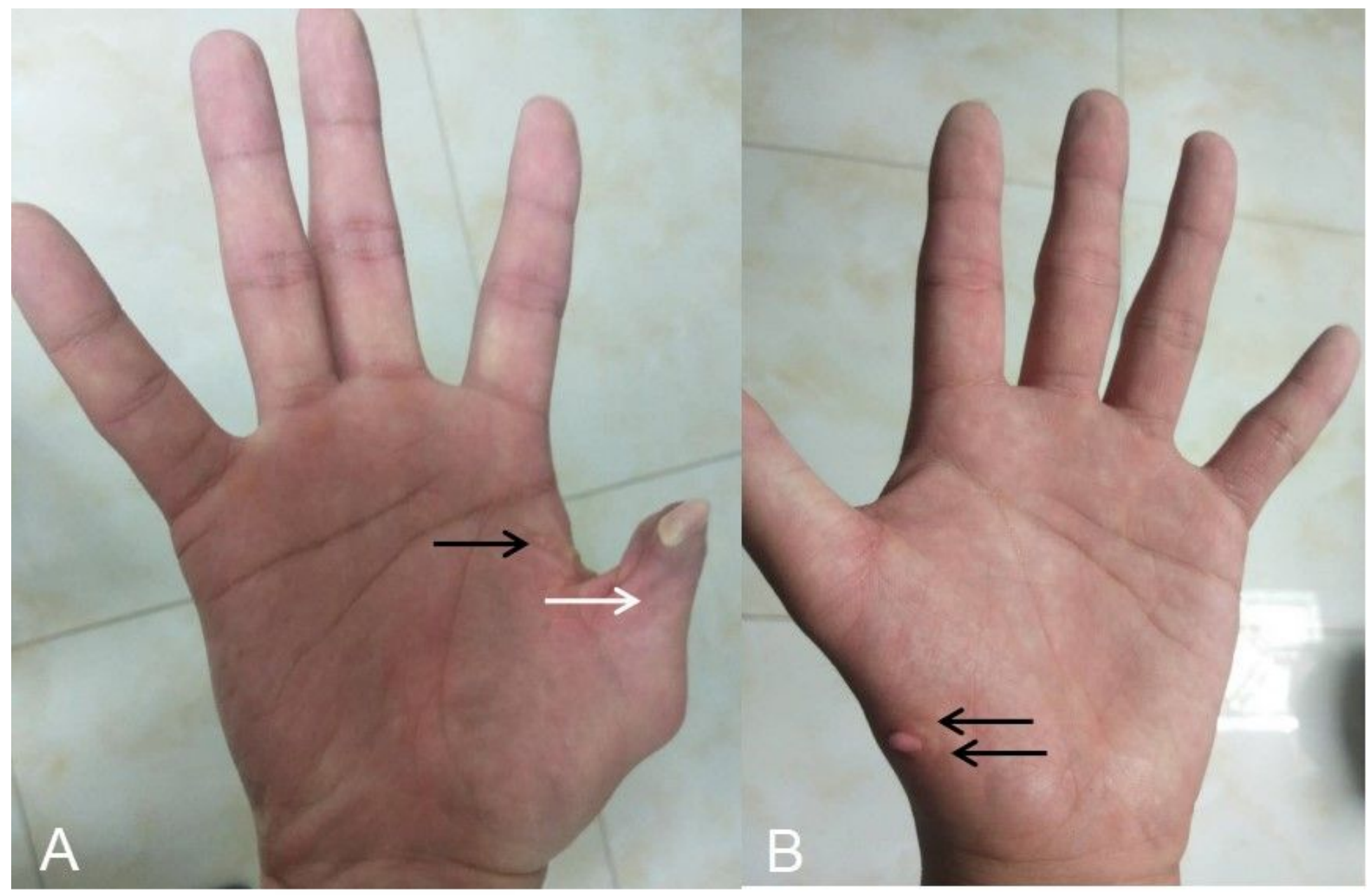

\section{Figure 1}

(A) The black arrow indicates the surgical scar from surplus finger resection. The white arrow indicates the small and deformed thumb. (B) The double black arrows indicate the small, convex tissue considered to be an incompletely developed finger. 


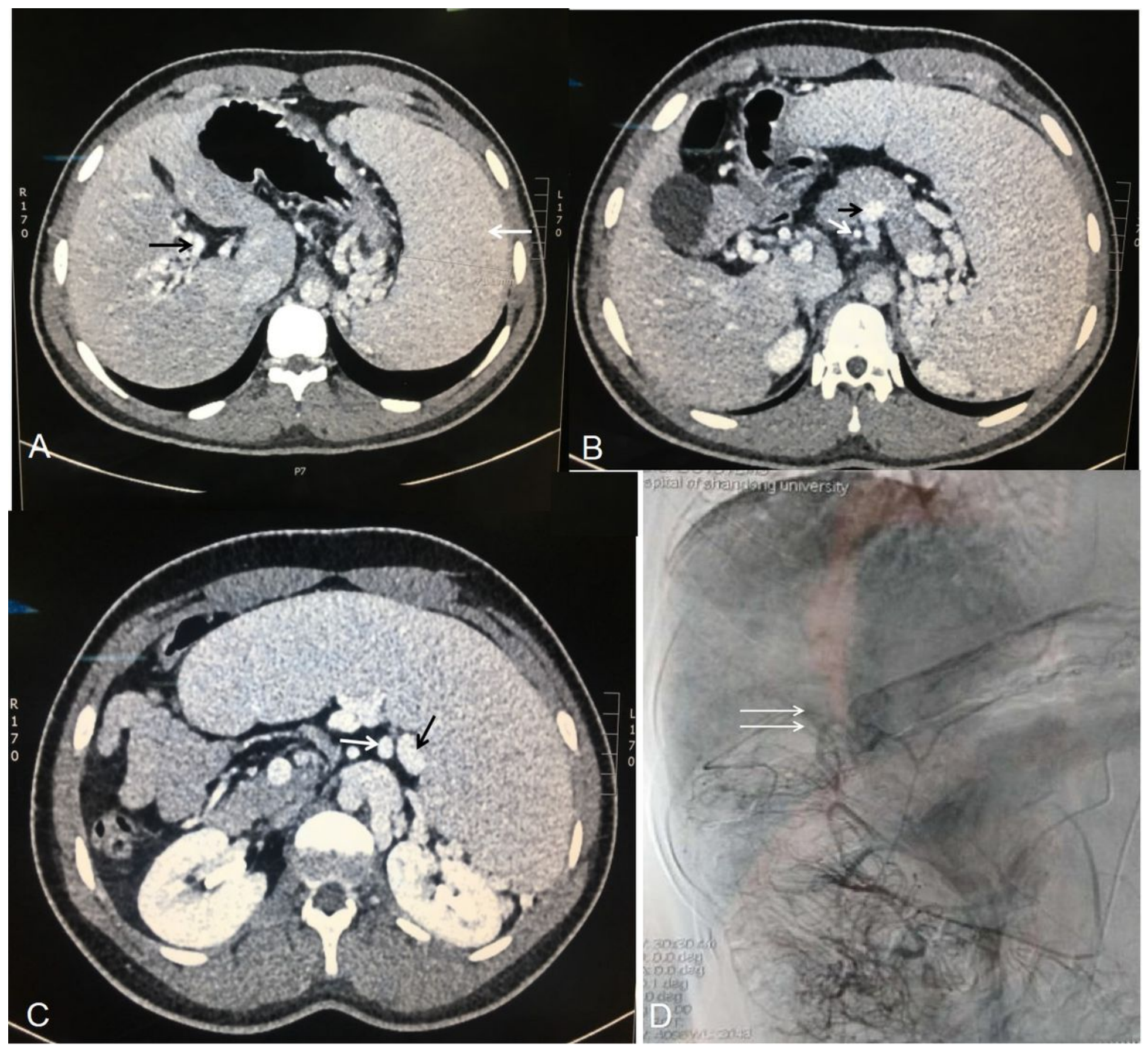

Figure 2

(A) The black arrow indicates the cavernous transformation of the portal vein. The white arrow indicates splenomegaly. (B) Anastomosis of the gastric fundus vein and splenic vein. The black arrow indicates the splenic vein. The white arrow indicates one of the gastric fundus veins. (C) Anastomosis of the left renal vein and splenic vein. The black arrow indicates the splenic vein. The white arrow indicates the left renal vein. (D) The double white arrows indicate the contrast medium observed in the portal vein closed to the splenic vein, instead of intrahepatic segment in the delayed phase of indirect portography examination. 